\title{
Gas-liquid critical point of the ultrasoft restricted primitive model from analytic theory
}

\author{
O. Patsahan \\ Institute for Condensed Matter Physics of the National Academy of Sciences of Ukraine, \\ 1 Svientsitskii St., 79011 Lviv, Ukraine
}

Received April 22, 2015

\begin{abstract}
Gas-liquid criticality in the ultrasoft restricted primitive model (URPM) of polyelectrolytes is studied using the collective variables-based theory. For the model, an effective Hamiltonian is derived and explicit expressions for all the coefficients are found in a one-loop approximation. Based on this Hamiltonian, the phase and critical behaviour is analysed. Our results provide evidence that the nature of the gas-liquid criticality in the URPM is the same as in the restricted primitive model that includes a hard core.
\end{abstract}

Key words: polyelectrolytes, ultrasoft restricted primitive model, gas-liquid critical point, effective Hamiltonian

PACS: 64.75.-g, 64.60.De, 64.75.Cd, 64.60.fd

Recently, a new model of polyelectrolyte solutions, the so-called ultrasoft restricted primitive model (URPM), has been introduced in [1,2]. The URPM is a globally electroneutral equimolar mixture of positive and negative spatially extended charge distributions. Contrary to the familiar restricted primitive model (RPM) [3], no hard cores are involved in the URPM. Nevertheless, the URPM admits a thermodynamic limit since it is H-stable in the sence of Fisher and Ruelle [4, 5]. The phase diagram of the model was studied in [1, 2, 6] using Monte Carlo (MC) and molecular dynamic simulations. The analysis of simulation data for relatively small system sizes revealed a nontrivial topology of the phase diagram: a region of low temperature gas-liquid phase coexistence terminating at a critical point and a sharp conductor-insulator transition above this point [1, 2]. The simulation data also hinted at a tricritical nature of the gas-liquid critical point. Such a phase behaviour qualitatively differs from that found in the RPM. More recently [6], the gas-liquid phase transition and the critical behavior of the URPM have been studied using finite size scaling MC simulations in the grand canonical ensemble. While these simulation results confirm the existence of the gas-liquid critical point, they fail to provide a conclusive evidence for the nature of criticality of the model as well as reliable values for the critical exponents. In addition, the estimates of the critical point parameters $\left(T_{\mathrm{c}}^{*}=0.0134\right.$ and $\rho_{\mathrm{c}}^{*}=0.26$ ) differ from those given in [2], i.e., $T_{\mathrm{c}}^{*} \sim 0.018$, $\rho_{\mathrm{c}}^{*} \sim 0.16$ (expressed in the same reduced units as in [6]) which are obtained for smaller system sizes and without finite scaling size analysis. In general, the simulation results show a strong dependence of the coexistence envelope (its location and shape) on the system size compared to the case of the RPM. The gas-liquid phase coexistence in the URPM has been also predicted by the mean-field (MF) like theories [7, 8], although with the critical point being considerably distant from the simulations. As expected, the MF theories predict a classical critical behavior near the critical point.

Motivated by the above mentioned simulation studies, we address the issue of the gas-liquid criticality in the URPM using the theory that exploits the method of collective variables (CVs) [9, 10]. The theory enables us to derive, on the microscopic grounds, the effective Hamiltonian and find all the relevant coefficients, including the square-gradient term, within the framework of the same approximation [11]. Using this theory, we obtained the effective Hamiltonian of the RPM in the vicinity of the gas-liquid critical point that takes into account the contribution from higher order correlations between the positive and negative ions [11, 12]. The resulting form of this Hamiltonian confirms the fact that its critical behaviour 
belongs to the universal class of a three-dimensional Ising model. Herein below, we derive the effective Hamiltonian of the URPM and, on this basis, analyse the phase and critical behaviour.

We consider an equimolar mixture of $N_{+}$polycations of charge $+Q$ and $N_{-}\left(=N_{+}\right)$polyanions of charge $-Q$ in a volume $V$ with the total number density $\rho=N / V\left(N=N_{+}+N_{-}\right)$. The domain of the volume $V$ occupied by polyions is denoted by $\Omega$. Polyions bear an extended charge distribution $\pm Q \rho(r)$ where the normalized distribution $\rho(r)$ is supposed to be the same for both species and is given by a Gaussian law

$$
\rho(r)=\frac{\exp \left[-r^{2} /\left(2 \bar{\sigma}^{2}\right)\right]}{\left(2 \pi \bar{\sigma}^{2}\right)^{3 / 2}}
$$

$\bar{\sigma}=\sigma / 2$ is the radius of the polyion. The interaction potential between a polyion of species $\alpha$ and one of the species $\beta$ is as follows [2]:

$$
u_{\alpha \beta}(r)=\frac{Q_{\alpha} Q_{\beta}}{r} \operatorname{erf}(r / 2 \bar{\sigma}) .
$$

This pair potential is finite at a full overlap [erf $(r / 2 \bar{\sigma}) \sim r$ as $r \rightarrow 0$ ] ensuring the existence of the thermodynamic limit [4-6]. The Fourier transform of $u_{\alpha \beta}(r)$ has the form:

$$
\tilde{u}_{\alpha \beta}(k)=\frac{4 \pi Q_{\alpha} Q_{\beta}}{k^{2}} \exp \left(-k^{2} \bar{\sigma}^{2}\right) .
$$

The model (1) is at equilibrium in the grand canonical ensemble, and by $\beta=1 / k_{\mathrm{B}} T$ we denote the inverse temperature ( $k_{\mathrm{B}}$ is the Boltzmann constant) and $\mu$ is the chemical potential. We use the method of CVs, with the ideal gas system being a reference system. In this case, one can use the exact functional representation of the grand partition function derived for a multicomponent system in [13] (see equation (18) in [13]). For the model (1), it can be presented as follows:

$$
\Xi=\int \mathscr{D} \rho_{N} \int \mathscr{D} \rho_{Q} \exp \left(-\mathscr{H}\left[\rho_{N}, \rho_{Q}\right]\right) \text {, }
$$

where the Hamiltonian $\mathscr{H}\left[\rho_{N}, \rho_{Q}\right]$ has the form:

$$
\begin{aligned}
\mathscr{H}\left[\rho_{N}, \rho_{Q}\right]= & -\int \mathrm{d} \mathbf{r} \rho_{N}(\mathbf{r})+\frac{1}{2} \int \mathrm{d} \mathbf{r}\left[\rho_{N}(\mathbf{r})+\rho_{Q}(\mathbf{r})\right] \ln \left[\frac{\rho_{N}(\mathbf{r})+\rho_{Q}(\mathbf{r})}{2}\right] \\
& +\frac{1}{2} \int \mathrm{d} \mathbf{r}\left[\rho_{N}(\mathbf{r})-\rho_{Q}(\mathbf{r})\right] \ln \left[\frac{\rho_{N}(\mathbf{r})-\rho_{Q}(\mathbf{r})}{2}\right]+\frac{\beta}{2} \int \mathrm{d} \mathbf{r} \mathrm{d} \mathbf{r}^{\prime} u_{Q Q}\left(\left|\mathbf{r}-\mathbf{r}^{\prime}\right|\right) \\
& \times \rho_{Q}(\mathbf{r}) \rho_{Q}\left(\mathbf{r}^{\prime}\right)-\int \mathrm{d} \mathbf{r} \rho_{N}(\mathbf{r}) \bar{v}_{N} .
\end{aligned}
$$

In the above equations, $\rho_{N}$ and $\rho_{Q}$ denote the two CVs: $\rho_{N}(\mathbf{r})=\rho_{+}(\mathbf{r})+\rho_{-}(\mathbf{r})$ describing the field of the total number density and $\rho_{Q}(\mathbf{r})=\rho_{+}(\mathbf{r})-\rho_{-}(\mathbf{r})$ describing the field of the charge density $\left(\rho_{+(-)}(\mathbf{r})\right.$, in turn, describes the field of the number density of the cations (anions)). $u_{Q Q}$ is a linear combination of the interaction potentials $u_{\alpha \beta}(r): u_{Q Q}(r)=\frac{1}{4}\left[u_{++}(r)-2 u_{+-}(r)+u_{--}(r)\right] . \bar{v}_{N}=\left(\bar{v}_{+}+\bar{v}_{-}\right) / 2$, where $\bar{v}_{\alpha}(\mathbf{r})=v_{\alpha}+v_{\alpha}^{S}$ is the dimensionless chemical potential of the $\alpha$ th species, $v_{\alpha}=\beta \mu_{\alpha}-3 \ln \Lambda_{\alpha}, \Lambda_{\alpha}$ is the de Broglie thermal wavelength and $v_{\alpha}^{S}$ is the self-energy of the $\alpha$ th species $v_{\alpha}^{S}=\beta u_{\alpha \alpha}(\mathbf{r}, \mathbf{r}) / 2=\beta u_{0} / 2$ with $u_{0}=Q^{2} /(\sqrt{\pi} \bar{\sigma})$. For the model under consideration, $u_{Q Q}(r)=u_{+_{+}}(r)=u_{--}(r)=\left|u_{+_{-}}(r)\right|$ and $\bar{v}_{N}=\bar{v}_{+}=\bar{v}_{-}=\bar{v}$.

Functional integrals (3)-(4) can be given a precise meaning in the case where the domain $\Omega$ is a cube of side $L$ ( $V=L^{3}$ ) with periodic boundary conditions. This means that we restrict ourselves to the fields $\rho_{A}(\mathbf{r})(A=N, Q)$ which can be written as Fourier series $\rho_{A}(\mathbf{r})=V^{-1} \sum_{\mathbf{k} \in \Lambda} \rho_{\mathbf{k}, A} \exp (\mathrm{ikr})$, where $\Lambda=$ $(2 \pi / L) \mathbb{Z}^{3}$ is the reciprocal cubic lattice [14, 15].

Expanding the entropic part of Hamiltonian (4) in powers of $\rho_{N}$ and $\rho_{Q}$ (more exactly, in powers of deviations of $\rho_{N}$ and $\rho_{Q}$ from their MF values), we arrive at the expression similar to that obtained in [11] (see equation (7) in [11]). The main difference is that in the case of the URPM, the contributions from the hard sphere reference system to series expansion coefficients $a_{n}^{\left(i_{n}\right)}$ reduce to the ideal gas terms. Next, following the procedure developed in [11], one can derive the effective Hamiltonian of the URPM near the gas-liquid critical point. Here, we directly use the expressions obtained in [11] replacing the 
contributions from the hard sphere system with the corresponding terms for the ideal gas model. As a result, the effective Hamiltonian in the Fourier space representation reads as

$$
\begin{aligned}
\mathscr{H}^{\mathrm{eff}}= & a_{1,0} \rho_{0, N}+\frac{1}{2 !\langle N\rangle} \sum_{\mathbf{k}}\left(a_{2,0}+k^{2} a_{2,2}\right) \rho_{\mathbf{k}, N} \rho_{-\mathbf{k}, N} \\
& +\sum_{n \geqslant 3} \frac{1}{n !\langle N\rangle^{n-1}} \sum_{\mathbf{k}_{1}, \ldots, \mathbf{k}_{n}} a_{n, 0} \rho_{\mathbf{k}_{1}, N} \rho_{\mathbf{k}_{2}, N} \ldots \rho_{\mathbf{k}_{\mathbf{n}}, N} \delta_{\mathbf{k}_{1}+\ldots+\mathbf{k}_{n}},
\end{aligned}
$$

where $\delta_{\mathbf{k}_{1}+\ldots+\mathbf{k}_{n}}$ is the Kronecker symbol. The coefficients in a one-loop approximation have the form:

$$
\begin{aligned}
& a_{1,0}=-\Delta v_{N}-\frac{1}{2\langle N\rangle} \sum_{\mathbf{q}} \widetilde{g}_{Q}(q), \\
& a_{n, 0}=(-1)^{n}(n-2) !-\frac{(n-1) !}{2\langle N\rangle} \sum_{\mathbf{q}}\left[\widetilde{g}_{Q}(q)\right]^{n}, \quad n \geqslant 2, \\
& a_{2,2}=-\frac{1}{4\langle N\rangle} \sum_{\mathbf{q}} \widetilde{g}_{Q}^{(2)}(q)\left[1+\widetilde{g}_{Q}(q)\right],
\end{aligned}
$$

where $\Delta v_{N}=\bar{v}_{N}-v_{N}^{\text {id }}$, the superscript “id” refers to the ideal gas system. $\widetilde{g}_{Q}(q)$ is the screened potential

$$
\widetilde{g}_{Q}(q)=-\frac{\beta \rho \widetilde{u}_{Q Q}(q)}{1+\beta \rho \widetilde{u}_{Q Q}(q)}
$$

and the superscript (2) in equation (8) denotes the second-order derivative of $\widetilde{g}_{Q}(q)$ with respect to the wave vector. The terms involving screened potentials arise from integration in (3) over the charge subsystem $\left(\operatorname{CVs} \rho_{Q}\right)$.

Taking into account (2) and (9), one can obtain explicit expressions for coefficients (6)-(8):

$$
\begin{aligned}
& a_{1,0}=-v+v^{\text {id }}-\frac{1}{2 T^{*}}+\frac{1}{\sqrt{\pi}} \int_{0}^{\infty} \frac{x^{2} \mathrm{~d} x}{T^{*} x^{2} e^{x^{2}+24 \eta \sqrt{\pi}}},
\end{aligned}
$$

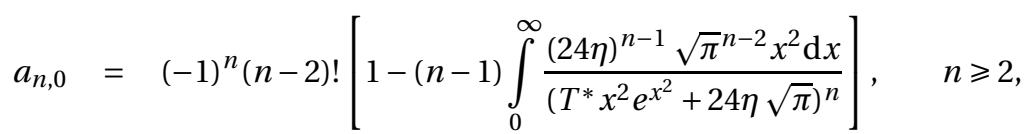

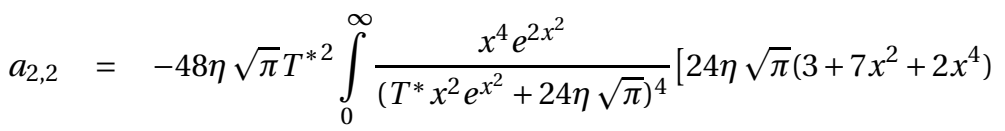

$$
\begin{aligned}
& \left.-T^{*} x^{2} e^{x^{2}}\left(1+x^{2}+2 x^{4}\right)\right] \mathrm{d} x .
\end{aligned}
$$

In equations (10)-(12), the following notations are introduced: $T^{*}=k_{\mathrm{B}} T / u_{0}=k_{\mathrm{B}} T \sqrt{\pi} \bar{\sigma} / Q^{2}, \eta=\pi \rho \bar{\sigma}^{3} / 6$, and $x=k \bar{\sigma}$. Coefficient $a_{1,0}$ is the excess part of the chemical potential $v$, and the equation $a_{1,0}=0$ yields the chemical potential in the random phase approximation (RPA).

It should be emphasized that although the original Hamiltonian of the URPM [equation (4)] does not include direct pair attractive interactions of total number density fluctuations, the effective short-range attraction does appear in the effective Hamiltonian. This attractive interaction is the result of taking into account the charge-charge correlations through integration over the charge subsystem. Therefore, one can state that the mechanism of the gas-liquid separation in the URPM is the same as in the RPM. The form of the effective Hamiltonian (5)-(8) suggests the Ising-like critical behaviour of the URPM. Herein below, we use explicit expressions (10)-12) for the analysis of the gas-liquid phase separation.

First, we consider the critical point. At the critical point, the system of equations

$$
a_{2,0}\left(\rho_{\mathrm{c}}, T_{\mathrm{c}}\right)=0, \quad a_{3,0}\left(\rho_{\mathrm{c}}, T_{\mathrm{c}}\right)=0
$$

holds. In addition to equations (13), we solve the equation $a_{4,0}=0$. The loci of the above-mentioned equations in the $\left(T^{*}, \rho^{*}\right)$ plane are shown in figure 1 The solid line corresponding to the solutions of the 


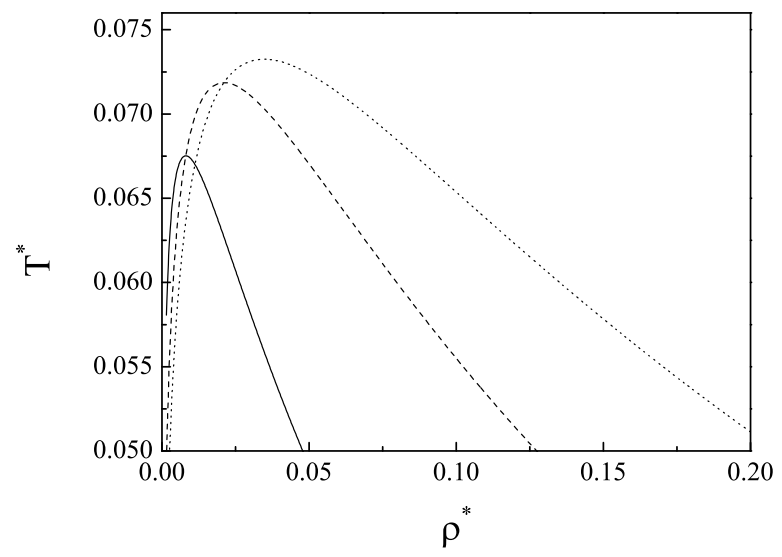

Figure 1. Loci of equations $a_{2,0}=0$ (solid line), $a_{3,0}=0$ (dashed line), and $a_{4,0}=0$ (dotted line). Temperature and density are given in dimensionless unites: $T^{*}=k_{\mathrm{B}} T \sqrt{\pi} \sigma /\left(2 Q^{2}\right)$ and $\rho^{*}=\rho \sigma^{3}$.

first equation in (13) is called a spinodal line. The two lines (solid and dashed) intersect at a maximum of the gas-liquid spinodal yielding the coordinates of the critical point. As is seen, coefficient $a_{4,0}$ does not vanish at the critical point suggesting that this point is a regular critical point.

Critical temperature and critical density expressed in reduced units are presented in table1 In order to make some contact with the results obtained for the RPM, hereafter we use the same reduced units for the density as in [6], i.e., $\rho^{*}=\rho \sigma^{3}$ where $\sigma$ is the diameter of the polyion. Our choice of the reduced temperature, $T^{*}=k_{\mathrm{B}} T / u_{0}$ with $u_{0}$ being the maximum strength of the attractive interaction, coincides with that of [1, 2, 6, 7]. In particular, one gets $u_{0}=2 Q^{2} /(\sqrt{\pi} \sigma)$ for the URPM and $u_{0}=Q^{2} / \sigma$ in the case of the RPM where $\sigma$ is the diameter of the polyion in the former case and the diameter of the hard sphere/ion in the latter case. With the critical parameters defined in such a way, the critical temperature of the URPM appears to be smaller than the critical temperature of the RPM obtained in the same approximation, whereas the critical densities of the both models are nearly the same. As expected, our estimates of the critical parameters of the URPM coincide with the results obtained in [8] in the RPA. It should be noted that the RPA, like other mean-field theories [7], predicts a far too high critical temperature and a far too low critical density compared with the available simulation data [1, 2, 6]. Some possible reasons for such a situation have been discussed in [7].

Table 1. Reduced gas-liquid critical parameters, coefficients of the effective Hamiltonian and the reduced Ginzburg temperature $t_{\mathrm{G}}$ for the ultrasoft restricted primitive model (URPM) and for the restricted primitive model (RPM) in the one-loop approximation. Temperature and density are given in dimensionless unites: $T_{\mathrm{c}}^{*}=k_{\mathrm{B}} T_{\mathrm{c}} / u_{0}$ and $\rho_{\mathrm{c}}^{*}=\rho_{\mathrm{c}} \sigma^{3}$ (see the text for details). The data for the RPM are taken from [11].

\begin{tabular}{|c|c|c|c|c|c|c|}
\hline Model & $T_{\mathrm{c}}^{*}$ & $\rho_{\mathrm{c}}^{*}$ & $a_{2, t}$ & $a_{2,2} / \sigma^{2}$ & $a_{4,0}$ & $t_{\mathrm{G}}$ \\
\hline \hline URPM & 0.06750 & 0.0081 & 1.0005 & 0.2693 & 0.1384 & 0.0031 \\
RPM & 0.08446 & 0.0088 & 1.0758 & 0.2570 & 0.1752 & 0.0053 \\
\hline
\end{tabular}

To calculate the coexistence curve, first, equating the right-hand side of (10) to zero, we obtain an expression for the chemical potential. Then, the Maxwell double-tangent construction is employed. Figure 2 shows the coexistence curve of the URPM (a solid line) in the $\left(T^{*}, \rho^{*}\right)$ plane. The coexistence curve of the RPM (a dashed line) is presented for comparison. As is seen, the shape of the both curves is very similar. The inset shows the both coexistence curves in the corresponding-state variables: $t^{*}=\left(T^{*}-T_{\mathrm{c}}^{*}\right) / T_{\mathrm{c}}^{*}$ and $\Delta \rho^{*}=\left(\rho^{*}-\rho_{\mathrm{c}}^{*}\right) / \rho_{\mathrm{c}}^{*}$. Comparing the two coexistence envelopes in this case, one can see that the URPM has a slightly wider coexistence region than the RPM.

Finally, using equations (11) and (12), we calculate the reduced Ginzburg temperature $t_{\mathrm{G}}$ where $t_{\mathrm{G}}=$ $\left(T_{\mathrm{G}}-T_{\mathrm{C}}\right) / T_{\mathrm{c}}\left(T_{\mathrm{G}}\right.$ is the Ginzburg temperature and $T_{\mathrm{c}}$ is the MF critical temperature). According to the 
Ginzburg criterion [15], $t_{\mathrm{G}}$ marks a lower bound of the temperature region where a MF description is self-consistent. For $|t| \ll t_{\mathrm{G}}$, Ising critical behavior should be exhibited. One expects a crossover from the MF behaviour to the Ising-like critical behaviour when the reduced temperature $|t|$ becomes of the order of $t_{\mathrm{G}}$ [16]. The reduced Ginzburg temperature can be expressed in terms of coefficients of the Hamiltonian (5) as follows [17]:

$$
t_{\mathrm{G}}=\frac{1}{32 \pi^{2}} \frac{a_{4,0}^{2}}{a_{2, t} a_{2,2}^{3}},
$$

where $a_{2, t}=\partial a_{2,0} /\left.\partial t\right|_{t=0}$. Taking into account (11), one can obtain for $a_{2, t}$

$$
a_{2, t}=48 \eta T_{\mathrm{c}}^{*} \int_{0}^{\infty} \frac{x^{4} e^{x^{2}} \mathrm{~d} x}{\left(T_{\mathrm{c}}^{*} x^{2} e^{x^{2}}+24 \eta \sqrt{\pi}\right)^{3}}
$$

where the same dimensionless units as in (11) are used.

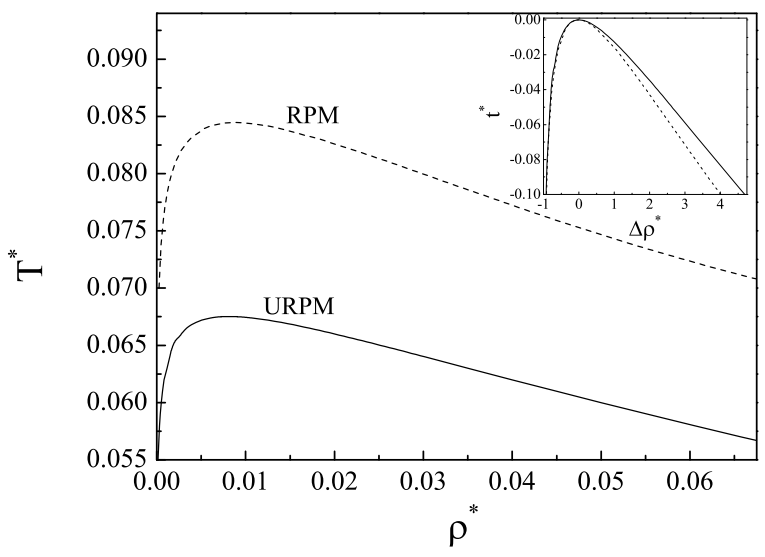

Figure 2. Gas-liquid coexistence curve of the URPM in the $\left(T^{*}, \rho^{*}\right)$ representation. The coexistence curve of the RPM (a dashed line) is presented for comparison (see the text for details). The inset shows the both coexistence curves in the corresponding-state variables.

Substituting the parameters of the URPM critical point in equations (11) (for $n=4$ ), (12) and (14)-(15), one gets the values for the Hamiltonian coefficients and the reduced Ginzburg temperature in the oneloop approximation. The results are presented in table 1, The corresponding values for the RPM obtained within the framework of the same approximation in [11] are presented in the table for comparison. As is seen, there is only a little difference between the results obtained for the both models. In particular, the models have much the same region of the effective density-density attractions: $\sqrt{a_{2,2}} / \sigma \simeq 0.5$. It is essential that the reduced Ginzburg temperature obtained for the URPM is close to that found for the $\mathrm{RPM}$, i.e., $t_{\mathrm{G}}^{\mathrm{URPM}} \simeq 0.6 t_{\mathrm{G}}^{\mathrm{RPM}}$. It is worth noting that the reduced Ginzburg temperature of the RPM is about 20 times smaller than for the purely nonionic model (a one-component hard-sphere square-well model) [11].

In summary, we have derived a microscopic-based effective Hamiltonian of the URPM in the vicinity of the gas-liquid critical point and have found analytic expressions for all the coefficients in the one-loop approximation. As for the RPM, the form of the effective Hamiltonian indicates that the critical behavior of the URPM belongs to the universal class of a three-dimensional Ising model. Based on this Hamiltonian, we have calculated the reduced Ginzburg temperature $t_{\mathrm{G}}$ which, according to the Ginzburg criterion, marks the onset of the critical region. We have found that $t_{\mathrm{G}}$ for the URPM is of the same order as for the RPM. In addition, our results have shown that the shapes of coexistence envelope of the URPM and the RPM resemble each other when presented in the similar dimensionless units. Our results suggest that the gas-liquid criticality in both models, i.e., the URPM and the RPM, should be of the same nature. 


\title{
References
}

1. Coslovich D., Hansen J.-P., Kahl G., Soft Matter, 2011, 7, 1690; doi 10.1039/C0SM01090A

2. Coslovich D., Hansen J.-P., Kahl G., J. Chem. Phys., 2011, 134, 244514; doi 10.1063/1.3602469

3. Hansen J.P., McDonald I.R., Theory of simple liquids, Academic Press, 1986.

4. Ruelle D., Statistical Mechanics, Rigorous Results, W.A. Benjamin, New York, 1969.

5. Fisher M.E., Ruelle D., J. Math. Phys., 1966, 7, 260; doi 10.1063/1.1704928

6. Caillol J.-M., Levesque D., J. Chem. Phys., 2014, 140, 214505; doi 10.1063/1.4880239

7. Nikoubashman A., Hansen J.-P., Kahl G., J. Chem. Phys., 2012, 137, 094905; doi 10.1063/1.4748378

8. Warren P.B., Masters A.J., J. Chem. Phys., 2013, 138, 074901; doi 10.1063/1.4791635

9. Yukhnovskii I.R., Holovko M.F., Statistical Theory of Classical Equilibrium Systems, Naukova Dumka, Kiev, 1980 (in Russian).

10. Patsahan O., Mryglod I., Condens. Matter Phys., 2006, 9, 659; doi 10.5488/CMP.9.4.659.

11. Patsahan O.V., Phys. Rev. E, 2013, 88, 022102; doi 10.1103/PhysRevE.88.022102

12. Patsahan O.V., Mryglod I.M., J. Phys.: Condens. Matter, 2004, 16, L235; doi 10.1088/0953-8984/16/16/L01

13. Patsahan O., Mryglod I., Condens. Matter Phys., 2012, 15, 24001; doi 10.5488/CMP.15.24001

14. Patsahan O., Mryglod I., Caillol J.-M., J. Phys. Stud., 2007, 11, 133.

15. Goldenfeld N., Lectures on Phase Transitions and the Renormalization Group, Addison-Wesley, New-York, 1992.

16. Chaikin P.M., Lubensky T.C., Principles of Condensed Matter Physics, Cambridge University Press, 1995.

17. Fisher M.E., Lee B.P., Phys. Rev. Lett., 1996, 77, 3561; doi 10.1103/PhysRevLett.77.3561

\section{Критична точка газ-рідина ультрам'якої обмеженої примітивної моделі: аналітична теорія}

\author{
О. Пацаган \\ Інститут фізики конденсованих систем НАН України, вул. Свєнціцького, 1, 79011 м. Львів, Україна
}

Вивчається критичність газ-рідина ультрам'якої обмеженої примітивної моделі (URPM) поліелектролітів, використовуючи теорію, що базується на методі колективних змінних. Для цієї моделі виведено ефективний гамільтоніан і отримано явні вирази для всіх коефіцієнтів в однопетлевому наближенні. На основі цього гамільтоніану аналізується фазова і критична поведінка. Отримані результати свідчать про те, що природа критичності газ-рідина в URPM $\epsilon$ такою ж, як і в обмеженій примітивній моделі, яка включає твердий кор.

Ключові слова: поліелектроліти, ультрам'яка обмежена примітивна модель, критична точка газ-рідина, ефективний гамільтоніан 